\title{
Guarded subgraphs and the domination game
}

\author{
Boštjan Brešar $\left.{ }^{1,2}\right|^{*}$ Sandi Klavžar ${ }^{1,2,3 \mid}$ Gasper Košmrlj 非 Doug F. Rall非 \\ ${ }^{1}$ Faculty of Natural Sciences and Mathematics, University of Maribor, Slovenia \\ ${ }^{2}$ Institute of Mathematics, Physics and Mechanics, Ljubljana \\ ${ }^{3}$ Faculty of Mathematics and Physics, University of Ljubljana, Slovenia \\ ${ }^{4}$ Department of Mathematics, Furman University, Greenville, SC, USA
}

received $8^{\text {th }}$ June 2014, accepted $12^{\text {th }} \mathrm{Feb} .2015$.

\begin{abstract}
We introduce the concept of guarded subgraph of a graph, which as a condition lies between convex and 2-isometric subgraphs and is not comparable to isometric subgraphs. Some basic metric properties of guarded subgraphs are obtained, and then this concept is applied to the domination game. In this game two players, Dominator and Staller, alternate choosing vertices of a graph, one at a time, such that each chosen vertex enlarges the set of vertices dominated so far. The aim of Dominator is that the graph is dominated in as few steps as possible, while the aim of Staller is just the opposite. The game domination number is the number of vertices chosen when Dominator starts the game and both players play optimally. The main result of this paper is that the game domination number of a graph is not smaller than the game domination number of any guarded subgraph. Several applications of this result are presented.
\end{abstract}

Keywords: domination game; game domination number; convex subgraph; (2-)isometric subgraph

\section{Introduction and preliminaries}

Graph domination with its many variations is one of the most studied topics in graph theory; it has been surveyed in a monograph in 1998 [8]. Recall that a set $D \subseteq V(G)$ is dominating if every vertex from $V(G)-D$ has a neighbor in $D$. The minimum size of a dominating set of a graph $G$ is called the domination number of $G$, denoted $\gamma(G)$. Note that the domination number of $G$ can either be bigger or smaller than the domination number of an induced subgraph. For the former consider for instance a clique in a graph that has an arbitrarily large domination number. For the latter let $H$ have large domination number and construct $G$ by adding a universal vertex.

In this paper we define a type of subgraph that we call guarded, for which the domination number is hereditary. Moreover, this is also proven for the two game counterparts of domination, namely the game domination number and the Staller-start game domination number. As it turns out, guarded subgraphs

\footnotetext{
*Email: bostjan.bresar@um.si

†Email: sandi.klavzarefmf.uni-lj.si

‡Email: gasper.kosmrljestudent.fmf.uni-lj.si

§Email: doug.rallefurman.edu

1365-8050 @ 2015 Discrete Mathematics and Theoretical Computer Science (DMTCS), Nancy, France
} 
are interesting also from the metric point of view. Notably every convex subgraph is guarded, and every guarded subgraph is 2-isometric. (See [9, 14] for more about metric concepts in graphs.)

The domination game was introduced in [2], and studied in several papers [1, 3, 4, 5, 6, 7, 10, 11, 12, 15]. We consider two games played by Dominator and Staller in a graph $G$, where, in addition we sometimes assume that some subset $S$ of vertices is already dominated (more precisely, the vertices of $S$ may be played during the game, but need not be dominated). We denote the graph $G$ with $S$ as described above by $G \mid S$. In particular, if $S=\{x\}$ we will write $G \mid x$, and if $S=\emptyset$ we will write $G \mid \emptyset$ simply as $G$. By Game 1 we mean a game in which Dominator has the first move, while Game 2 refers to a game in which Staller begins. Recall that players alternate turns and may only choose a vertex $v$ for which $N[v]$ contains a vertex that was not previously dominated (a move with this property is called legal). Assuming that both players play optimally on $G \mid S$, the game domination number $\gamma_{g}(G \mid S)$, respectively the Staller-start game domination number $\gamma_{g}^{\prime}(G \mid S)$, denotes the number of moves played, equivalently the number of vertices chosen, in Game 1, respectively Game 2.

The Staller-pass game is the domination game in which, on each turn, Staller may pass her move. Let $\hat{\gamma}_{g}(G \mid S)$ be the number of moves in such a game played optimally on $G \mid S$ when Dominator starts, and $\hat{\gamma}_{g}^{\prime}(G \mid S)$ when Staller starts. The turns when Staller passes do not count as moves. With these concepts in hand we now recall several very useful results due to Kinnersley, West, and Zamani [10].

Lemma 1.1 (Continuation Principle) [10, Lemma 2.1] Let $G$ be a graph and $A, B \subseteq V(G)$. If $B \subseteq A$, then $\gamma_{g}(G \mid A) \leq \gamma_{g}(G \mid B)$ and $\gamma_{g}^{\prime}(G \mid A) \leq \gamma_{g}^{\prime}(G \mid B)$.

Lemma 1.2 (Segmentation Lemma) [10, Lemma 2.4] Let $G_{1} \mid A_{1}$ and $G_{2} \mid A_{2}$ be partially-dominated graphs. If $G=G_{1} \cup G_{2}$, then $\hat{\gamma}_{g}\left(G \mid\left(A_{1} \cup A_{2}\right)\right) \leq \hat{\gamma}_{g}\left(G_{1} \mid A_{1}\right)+\hat{\gamma}_{g}^{\prime}\left(G_{2} \mid A_{2}\right)$ and $\hat{\gamma}_{g}^{\prime}\left(G \mid\left(A_{1} \cup A_{2}\right)\right) \leq$ $\hat{\gamma}_{g}^{\prime}\left(G_{1} \mid A_{1}\right)+\hat{\gamma}_{g}^{\prime}\left(G_{2} \mid A_{2}\right)$.

Theorem 1.3 [10, Theorem 4.6] If $F$ is a forest and $S \subseteq V(F)$, then $\gamma_{g}(F \mid S) \leq \gamma_{g}^{\prime}(F \mid S)$.

Lemma 1.4 [10, Corollary 4.7] If $F$ is a forest and $S \subseteq V(F)$, then $\hat{\gamma}_{g}(F \mid S)=\gamma_{g}(F \mid S)$ and $\hat{\gamma}_{g}^{\prime}(F \mid S)=$ $\gamma_{g}^{\prime}(F \mid S)$.

In addition, we recall the following result from [11].

Theorem 1.5 For any $n \geq 3$,

$$
\gamma_{g}\left(P_{n}\right)=\gamma_{g}\left(C_{n}\right)= \begin{cases}\left\lceil\frac{n}{2}\right\rceil-1 ; & n \equiv 3 \bmod 4 \\ \left\lceil\frac{n}{2}\right\rceil ; & \text { otherwise }\end{cases}
$$

The paper is organized as follows. In Section 2 we introduce and study guarded subgraphs from the metric point of view; we prove that every convex subgraph is guarded and every guarded subgraph is 2isometric. We also specify some conditions under which guarded and 2-isometric subgraphs coincide. In Section 3 we prove our main results that connect guarded subgraphs and domination. Notably, the (game) domination number of a graph is not smaller than the (game) domination number of a guarded subgraph. Then in Section 4 we present several applications of these theorems in the domination game.

\section{Guarded subgraphs}

In this section we introduce the concept of a guarded subgraph and deduce some of its properties to be used later. 
We say that a subgraph $H$ of a graph $G$ is guarded in $G$ if for any vertex $x$ in $G$ there exists a vertex $y \in V(H)$ such that $N[x] \cap V(H) \subseteq N[y] \cap V(H)$. Such a vertex $y$ will be called a guard of $x$ in $H$. Note that if $x \in V(H)$, then $x$ is a guard of itself. Observe also that if $x \notin V(H)$ and $|N[x] \cap V(H)|=1$, then the neighbor of $x$ in $H$ is a guard of $x$ in $H$.

Guarded subgraphs are closely related to several central concepts from metric graph theory. Recall that a subgraph $H$ of $G$ is isometric if $d_{H}(u, v)=d_{G}(u, v)$ holds for every pair of vertices $u$ and $v$ of $H$ and that $H$ is 2-isometric if for $d_{G}(u, v)=2, u, v \in V(H)$, it follows that $d_{H}(u, v)=2$. Clearly, an isometric subgraph is 2-isometric, but not the other way around. Recall also that a subgraph $H$ of $G$ is convex if for every pair of vertices $u$ and $v$ of $H$, every shortest $u, v$-path in $G$ lies in $H$. A convex subgraph is necessarily isometric. The next result places guarded subgraphs into this framework.

Proposition 2.1 A convex subgraph is guarded, and a guarded subgraph is 2-isometric.

Proof: Let $H$ be a convex subgraph of a graph $G$, let $u \in V(G) \backslash V(H)$ and let $S=N[u] \cap V(H)$. If $x, y \in S$, then $d_{G}(x, y) \leq 2$ and since $H$ is convex $x$ must be adjacent to $y$. It follows that $S$ induces a complete subgraph of $H$ and thus any vertex of $S$ is a guard of $u$ in $H$. This proves the first implication.

Assume now that $H$ is guarded in $G$ and let $u$ and $v$ be vertices of $H$ with $d_{G}(u, v)=2$. This means there is a vertex $x$ in $G$ that is a common neighbor of $u$ and $v$. If $x \in V(H)$, then $d_{H}(u, v)=2$. Otherwise, since $H$ is guarded in $G$, there exists a vertex $y$ in $H$ such that $N[x] \cap V(H) \subseteq N[y] \cap V(H)$. Note that $y \neq u$ and $y \neq v$ or else $d_{G}(u, v)=1$. It then follows that $y \in V(H)$ is a common neighbor of $u$ and $v$ and hence $d_{H}(u, v)=2$. We conclude that $H$ is 2-isometric.

In Fig. 1 relations between convex, isometric, guarded, and 2-isometric subgraphs are given. For each of the possibilities an example subgraph is shown in bold.

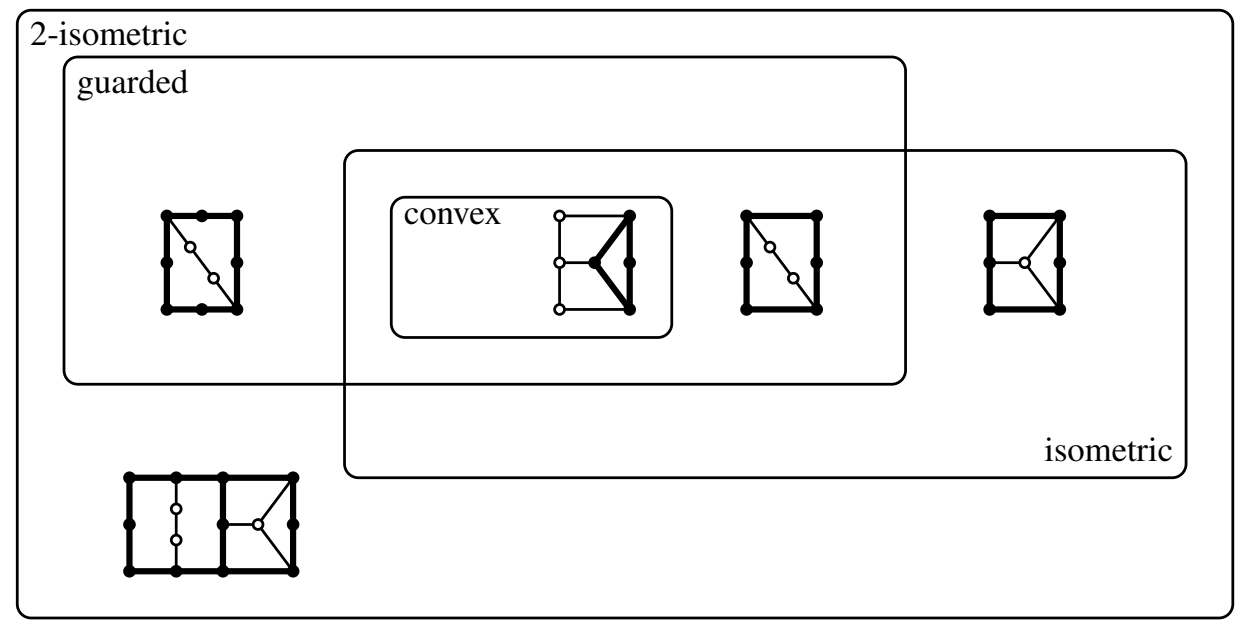

Fig. 1: Metric classes

In some special cases guarded subgraphs coincide with 2-isometric subgraphs. To make this more precise we let

$$
\Delta(G \mid H)=\max \{|N(x) \cap V(H)|: x \in V(G) \backslash V(H)\} .
$$


Then we have:

Proposition 2.2 Let $H$ be a subgraph of $G$.

(i) If $\Delta(G \mid H) \leq 2$, then $H$ is guarded in $G$ if and only if $H$ is 2-isometric in $G$.

(ii) If $\Delta(G \mid H) \leq 3$ and $G$ is a $\left\{K_{3}, C_{6}\right\}$-free graph, then $H$ is guarded in $G$ if and only if $H$ is 2-isometric in $G$.

Proof: (i) Suppose that $\Delta(G \mid H) \leq 2$ and that $H$ is 2-isometric in $G$. Let $x$ be a vertex from $V(G) \backslash V(H)$ that has two neighbors $u$ and $v$ in $V(H)$. If $u v \in E(G)$, then $N[x] \cap V(H)=\{u, v\} \subseteq N[u] \cap V(H)$. If $u v \notin E(G)$, then because $H$ is 2-isometric there exists a vertex $y$ in $H$ that is adjacent to both of $u$ and $v$. Hence, $H$ is guarded in $G$. The converse follows by Proposition 2.1 .

(ii) Let $x \in V(G) \backslash V(H)$. The case when $x$ has at most two neighbors in $H$ is covered by the first part of the proposition. Hence let $N(x) \cap V(H)=\{u, v, w\}$. Using the fact that $H$ is a 2-isometric subgraph of $G$ and that $G$ is $K_{3}$-free, each pair of this triple has a common neighbor in $H$. If at least two of these common neighbors are the same vertex, then this vertex is a guard for $x$. Otherwise, let $u^{\prime}, v^{\prime}$, and $w^{\prime}$ be the common neighbors of $v, w, u, w$, and $u, v$, respectively. Note that $\left\{u^{\prime}, v^{\prime}, w^{\prime}\right\}$ is an independent set since $G$ is $K_{3}$-free. Moreover, $u^{\prime}, v^{\prime}$ and $w^{\prime}$ are not adjacent to $u, v$ and $w$, respectively. It follows that $u, v^{\prime}, w, u^{\prime}, v, w^{\prime}$ induce a $C_{6}$ in $G$, the graph excluded with our assumption. Again, the converse follows by Proposition 2.1 .

\section{Guarded subgraphs in domination}

We begin with the following hereditary property of guarded subgraphs with respect to the domination number.

Proposition 3.1 If $H$ is guarded in $G$, then $\gamma(H) \leq \gamma(G)$.

Proof: Let $D_{G}$ be a minimum dominating set of $G$ and let $D_{G}^{\prime}=\left\{x \in D_{G} \cap(V(G) \backslash V(H))\right.$ : $N(x) \cap V(H) \neq \emptyset\}$. For each vertex $x \in D_{G}^{\prime}$, let $g(x)$ be an arbitrary but fixed guard of $x$ in $H$. Define $D_{H}=\left(D_{G} \cap V(H)\right) \cup\left\{g(x): x \in D_{G}^{\prime}\right\}$. Then $D_{H}$ is a dominating set and $\gamma(H) \leq\left|D_{H}\right| \leq\left|D_{G}\right|=$ $\gamma(G)$.

Let $H$ be a subgraph of a graph $G$. In general there is no relation between $\gamma_{g}(H)$ and $\gamma_{g}(G)$. Clearly, $\gamma_{g}(H)$ can be arbitrarily smaller than $\gamma_{g}(G)$. On the other hand, consider the wheel $W_{n}$ with $n$ spokes. It has a universal vertex $x$, hence $\gamma_{g}\left(W_{n}\right)=1$. The subgraph $H=G-x$ is isomorphic to $C_{n}$, hence $\gamma_{g}(H)$ can be also be arbitrarily larger than $\gamma_{g}(G)$. However, the latter phenomenon cannot happen for guarded subgraphs:

Theorem 3.2 If $H$ is guarded in $G$, then $\gamma_{g}(H) \leq \gamma_{g}(G)$ and $\gamma_{g}^{\prime}(H) \leq \gamma_{g}^{\prime}(G)$.

Proof: Let Game 1 be played in a graph $G$. We present a strategy of Staller that will enforce at least $\gamma_{g}(H)$ moves in the game. Along with the real game in $G$, Staller will imagine another Game 1 is being played in $H$. Each move of Dominator in the real game will be 'copied' by Staller into the imagined game in $H$, where Staller will respond optimally, and copy her move into the real game (the details will be presented along the way). The key property, ensured by the strategy, is the following: 
(P) After each move, made by either player, the set of vertices $S_{\text {real }}$ that belong to $H$ and are dominated in the real game, is a subset of $S_{\mathrm{im}}$, the set of vertices from $H$, dominated in the imagined game.

Property $(\mathrm{P})$ is clearly true in the beginning of the game, when $S_{\text {real }}=S_{\mathrm{im}}=\emptyset$. We proceed by induction on the number of moves in the game. Let $v$ be a vertex, chosen by Dominator at some point in the (real) game, and let $S_{\mathrm{im}}^{\prime}$ be the set of vertices from $H$, dominated before that move in the imagined game (similarly, $S_{\text {real }}^{\prime}$ denotes the set of vertices from $H$, dominated before that move in the real game). If $N[v] \cap\left(V(H) \backslash S_{\mathrm{im}}^{\prime}\right) \neq \emptyset$, then Staller imagines that Dominator played a guard of $v$ with respect to $G$. Note that this is a legal move of Dominator in the imagined game. In addition, $S_{\text {real }}$ is a subset of $S_{\mathrm{im}}$, since $S_{\text {real }}^{\prime} \subseteq S_{\mathrm{im}}^{\prime}$, hence $(\mathrm{P})$ is preserved after this move. On the other hand, if $N[v] \cap\left(V(H) \backslash S_{\mathrm{im}}^{\prime}\right)=\emptyset$, then an arbitrary vertex $w$ from $H$ is chosen by Staller for a move of Dominator in the imagined game, as long it is a legal move in this game. Again it is obvious that property $(\mathrm{P})$ is preserved after this move.

Now, when it is Staller's turn, she responds in the imagined game according to her optimal strategy that yields at least $\gamma_{g}(H)$ moves in this game. She copies her move to the real game (i.e., if her choice in the imagined game is to play a vertex $x$, then she plays $x$ also in the real game). Note that by the property (P) this move will be legal also in the real game, and it is clear that $(\mathrm{P})$ is preserved after her move, since the set $N[x] \cap V(H)$ is added (in the sense of set-union) to both sets $S_{\text {real }}$ and $S_{\text {im }}$. Now, since Staller is playing optimally in the imagined game, it will last at least $\gamma_{g}(H)$ steps. By (P), the real game will not end before the imagined game is over, hence the real game will also last at least $\gamma_{g}(H)$ steps. Since Staller was not necessarily playing optimally in the real game and Dominator was, we infer that the real game lasted at most $\gamma_{g}(G)$ moves. Thus, $\gamma_{g}(H) \leq \gamma_{g}(G)$.

Finally, when Game 2 is played in a graph $G$, the same strategy as in Game 1 can be used by Staller, from which we derive $\gamma_{g}^{\prime}(G) \leq \gamma_{g}^{\prime}(H)$.

Note that Theorem 3.2 cannot be extended by replacing the condition of $H$ being a guarded subgraph to the weaker condition of $H$ being a 2-isometric subgraph. Indeed, consider any graph $H$ with diameter 2 such that $\gamma_{g}(H)>1$, and let $G$ be obtained from $H$ by adding a vertex and make it adjacent to all vertices of $H$. Clearly, $H$ is (2-)isometric subgraph of $G$, but $\gamma_{g}(G)=1<\gamma_{g}(H)$. In fact, the difference $\gamma_{g}(H)-\gamma_{g}(G)$ can be arbitrarily large, as there are graphs $H$ with diameter 2 and (game) domination number arbitrarily large. For instance, letting $H$ be the Cartesian product $K_{n} \square K_{n}$ of the complete graph $K_{n}$ by itself, one gets $\operatorname{diam}(H)=2$ and $\gamma_{g}(H) \geq \gamma(H)=n$. (Recall that the Cartesian product $G \square H$ of graphs $G$ and $H$ has $V(G) \times V(H)$ as the vertex set, and two vertices are adjacent, whenever they coincide in one and are adjacent in the other coordinate.)

Theorem 3.2 can be applied when $G$ has several subgraphs that are pairwise at distance at least 3 , and each of the subgraphs is guarded in $G$. For this sake we recall the following result stated as the first assertion of Corollary 3.2 in [7].

Lemma 3.3 [7] If $G_{1} \mid S_{1}$ and $G_{2} \mid S_{2}$ are partially dominated graphs, then

$$
\gamma_{g}\left(\left(G_{1} \cup G_{2}\right) \mid\left(S_{1} \cup S_{2}\right)\right) \geq \gamma_{g}\left(G_{1} \mid S_{1}\right)+\gamma_{g}\left(G_{2} \mid S_{2}\right)-1 .
$$

Let now $H_{1}, \ldots, H_{m}$ be subgraphs of a graph $G$, each of which is guarded in $G$, such that $d\left(H_{i}, H_{j}\right)=$ $\min \left\{d(x, y): x \in V\left(H_{i}\right), y \in V\left(H_{j}\right)\right\} \geq 3$ whenever $1 \leq i<j \leq m$. By definition, the subgraph $H$ induced by $V\left(H_{1}\right) \cup \cdots \cup V\left(H_{m}\right)$ is also a guarded subgraph of $G$. As $H$ is the disjoint union of graphs $H_{1}, \ldots, H_{m}$, an inductive application of Lemma 3.3 (selecting $S_{1}$ and $S_{2}$ to be empty) yields

$$
\gamma_{g}(H) \geq \gamma_{g}\left(H_{1}\right)+\cdots+\gamma_{g}\left(H_{m}\right)-m+1 .
$$


Combining this with Theorem 3.2 we derive the following bound.

Corollary 3.4 Let $m \geq 1$. If $H_{1}, \ldots, H_{m}$ are guarded in $G$ and for any $i \neq j, d\left(H_{i}, H_{j}\right) \geq 3$, then

$$
\gamma_{g}(G) \geq \sum_{i=1}^{m} \gamma_{g}\left(H_{i}\right)-m+1
$$

To see that the bound of Corollary 3.4 is best possible let $G_{m}, m \geq 1$, be the graph constructed as follows. It consists of the disjoint union of $K_{m}$ and $m$ copies of $C_{6}$, denoted by $H_{i}, 1 \leq i \leq m$, where in addition the $i$ th vertex of $K_{m}$ is adjacent to three consecutive vertices of $H_{i}$. See Fig. 2 for $G_{4}$. Clearly, the subgraphs $H_{i}$ are pairwise at distance 3 and are guarded in $G_{m}$. It is now not difficult to observe that $\gamma_{g}\left(G_{m}\right)=2 m+1=\sum_{i=1}^{m} \gamma_{g}\left(C_{6}\right)-m+1$.

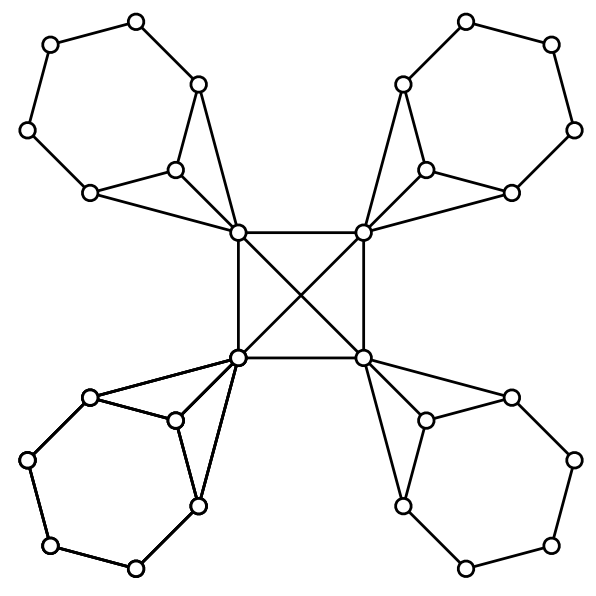

Fig. 2: The graph $G_{4}$ that attains the bound from Corollary 3.4

\section{Applications}

We start this section with some consequences of Theorem 3.2

Corollary 4.1 If $G$ is a connected graph, then

$$
\gamma_{g}(G) \geq\left\lfloor\frac{\operatorname{diam}(G)+1}{2}\right\rfloor .
$$

Proof: Let $H$ be a subgraph of $G$ induced by a diametrical path of $G$. Then $H$ is an isometric path. Let $x \in V(G) \backslash V(H)$ and let $u$ and $v$ be neighbors of $x$ in $H$. Since $d_{G}(u, v) \leq 2$ we also have $d_{H}(u, v) \leq 2$. If $d_{H}(u, v)=2$, then the vertex of $H$ adjacent to $u$ and $v$ is a guard of $x$ in $H$. It now readily follows that $H$ is guarded. Using Theorem 3.2 and Theorem 1.5 we get the result.

Corollary 4.1 should be compared with Theorem 2.24 from [8] asserting that $\gamma(G) \geq\left\lceil\frac{\operatorname{diam}(G)+1}{3}\right\rceil$ holds for any connected graph $G$. 
Corollary 4.2 If $G$ is a connected graph and $k$ is the length of a longest isometric cycle of $G$, then

$$
\gamma_{g}(G) \geq\left\lceil\frac{k}{2}\right\rceil-1
$$

The lower bounds of Corollaries 4.1 and 4.2 are in general incomparable. We also note that both bounds can be computed in polynomial time. This is a well-known fact for the diameter, while Lokshtanov [13] proved that a longest isometric cycle of a graph can be computed in polynomial time.

Let $H$ be a subgraph of $G$ and let $\partial H$ be the set of edges with one endpoint in $V(H)$ and the other in $V(G) \backslash V(H)$. The next result follows immediately from Theorem 3.2

Corollary 4.3 If $H$ is a subgraph of $G$ such that $\partial H$ is a matching, then $\gamma_{g}(H) \leq \gamma_{g}(G)$.

We continue with an application to trees.

Proposition 4.4 If $S$ is a tree and $T$ is the tree obtained from $S$ by adding a leaf adjacent to a vertex of $S$, then $\gamma_{g}(S) \leq \gamma_{g}(T) \leq \gamma_{g}(S)+1$.

Proof: The first inequality follows from Corollary 4.3 To prove the other inequality, let $y$ be the vertex of $S$ to which the leaf $x$ has been attached. Set $G_{1}=S$ and $G_{2}$ be the edge induced by $x$ and $y$. Then $T=G_{1} \cup G_{2}$ and by the Segmentation Lemma,

$$
\widehat{\gamma}_{g}(T) \leq \widehat{\gamma}_{g}\left(G_{1}\right)+\widehat{\gamma}_{g}^{\prime}\left(G_{2}\right)=\widehat{\gamma}_{g}(S)+1
$$

However Lemma 1.4 implies that $\widehat{\gamma}_{g}(T)=\gamma_{g}(T)$ and $\widehat{\gamma}_{g}(S)=\gamma_{g}(S)$, which establishes the second inequality.

A consequence of Proposition 4.4 is that given a tree $T$ one can recursively remove leaves and the resulting sequence of the game domination numbers is non-increasing and assumes all values between $\gamma_{g}(T)$ and 1 . This is not the case in general graphs. For instance consider the graph $G$ from Fig. 3 . The game domination number of $G$ is 5 , but $\gamma_{g}(G-u)=3$.

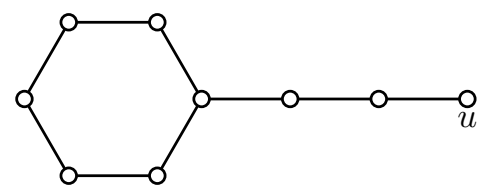

Fig. 3: The graph $G$.

Recall that the 2-packing number of a graph $G$ is the size of a largest subset $X$ of $V(G)$ such that $d_{G}(x, y) \geq 3$ holds for any different vertices $x$ and $y$ of $X$. Corollary 3.4 also gives an alternative proof of the following result.

Theorem 4.5 [2, Theorem 11] If $G$ and $H$ are arbitrary graphs, then

$$
\gamma_{g}(G \square H) \geq \rho(G)\left(\gamma_{g}(H)-1\right)+1 .
$$

With respect to the Cartesian product of graphs we also have the following result, where $G^{\square, n}$ denotes the $n$-tuple Cartesian product of $G$ and $Q_{n}$ the $n$-cube. 
Corollary 4.6 If $G$ is a connected graph and $n \geq 2$, then $\gamma_{g}\left(G^{\square, n}\right) \geq \gamma_{g}\left(G^{\square, n-1}\right)$. In particular, $\gamma_{g}\left(Q_{n}\right) \geq \gamma_{g}\left(Q_{n-1}\right)$.

Proof: Since $G^{\square, n}=G^{\square, n-1} \square G$, and $G^{\square, n-1}$ is a convex subgraph of $G^{\square, n}$ (cf. [9. Lemma 6.5]), the first assertion immediately follows from Theorem 3.2 and Proposition 2.1. For the second claim just recall that $Q_{n}=K_{2}^{\square, n}$.

\section{Acknowledgements}

We wish to thank an anonymous referee for the short proof of Corollary 3.4.

Research supported by a grant from the Simons Foundation (\#209654 to Douglas Rall) and by the Wylie Enrichment Fund of Furman University. B.B. and S.K. are supported by the Ministry of Science of Slovenia under the grant P1-0297. G.K. is also financed part by the European Union European Social Fund, and by Ministry of Economic Development and Technology of Republic of Slovenia under the grant 3211-11-000507.

\section{References}

[1] B. Brešar, P. Dorbec, S. Klavžar, G. Košmrlj, Domination game: Effect of edge- and vertex-removal, Discrete Math. 330 (2014) 1-10.

[2] B. Brešar, S. Klavžar, D. F. Rall, Domination game and an imagination strategy, SIAM J. Discrete Math. 24 (2010) 979-991.

[3] B. Brešar, S. Klavžar, D. F. Rall, Domination game played on trees and spanning subgraphs, Discrete Math. 313 (2013) 915-923.

[4] B. Brešar, S. Klavžar, G. Košmrlj, D. F. Rall, Domination game: extremal families of graphs for the 3/5conjectures, Discrete Appl. Math. 161 (2013) 1308-1316.

[5] Cs. Bujtás, Domination game on trees without leaves at distance four, Proceedings of the 8th JapaneseHungarian Symposium on Discrete Mathematics and Its Applications (A. Frank, A. Recski, G. Wiener, eds.), June 4-7, 2013, Veszprém, Hungary, 73-78.

[6] Cs. Bujtás, Domination game on forests, arXiv:1404.1382 [math.CO], 2014.

[7] P. Dorbec, G. Košmrlj, and G. Renault, The domination game played on unions of graphs, Discrete Math. 338 (2015) 71-79.

[8] T. W. Haynes, S. T. Hedetniemi, P. J. Slater, Fundamentals of Domination in Graphs, Marcel Dekker, New York, 1998.

[9] R. Hammack, W. Imrich and S. Klavžar, Handbook of Product Graphs, Second Edition, CRC Press, Boca Raton, FL, 2011.

[10] W. B. Kinnersley, D. B. West, R. Zamani, Extremal problems for game domination number, SIAM J. Discrete Math. 27 (2013) 2090-2107.

[11] W. B. Kinnersley, D. B. West, R. Zamani, Game domination for grid-like graphs, manuscript, 2012.

[12] G. Košmrlj, Realizations of the game domination number, J. Comb. Optim. 28 (2014) 447-461.

[13] D. Lokshtanov, Finding the longest isometric cycle in a graph, Discrete Appl. Math. 157 (2009) 2670-2674.

[14] I. M. Pelayo, Geodesic Convexity in Graphs, Springer, New York, 2013.

[15] H. G. Tananyan, Domination games played on line graphs of complete multipartite graphs, arXiv:1405.0087 [math.CO], 2014. 